\title{
Zukunft der intraoperativen Bildgebung und Navigation
}

\author{
Jochen Dick, Florian Gebhard
}

\section{Zusammenfassung}

Der Artikel befasst sich mit Technologien der Zukunft für die intraoperative Bildgebung und Navigation. Basierend auf dem Wandel im Gesundheitswesen und der medizinischen Versorgung werden Trends für die Chirurgie, wie z.B. die minimalinvasive Chirurgie oder das Qualitätsmanagement, abgeleitet und die Rolle der Bildgebung bez. dieser Trends erläutert. Im Weiteren werden die Limitierungen der heutigen Bildgebung und Navigation im chirurgischen Workflow erläutert und Technologien der Zukunft vorgestellt, welche zum Ziel haben, die intraoperative Bildgebung und Navigation besser in den chirurgischen Workflow zu integrieren als die heutigen Technologien. Zum Abschluss wird ein visionäres Szenario für den OP der Zukunft dargestellt.

\section{The Future of Intraoperative Imaging and Navigation}

This article addresses future technologies of intraoperative imaging and navigation. Based on changes in healthcare systems and medical care, trends in surgery, such as minimally invasive surgery or quality management, are derived. The role of imaging along these trends is elaborated. Furthermore, limitations of today's imaging and navigation technologies in the surgical workflow are discussed. Imaging and navigation technologies of the future are presented, which have the goal to improve integration into the surgical workflow. Finally a visionary scenario for the OR of the future is illustrated.

\section{Einleitung/Problemstellung}

Das Gesundheitswesen und damit die medizinische Versorgung sind aufgrund von demografischem Wandel, medizinischen und technologischen Fortschritten und den verbundenen sozioökonomischen Herausforderungen einem steten Wandel unterworfen. Daraus ergeben sich klinische und gesundheitsökonomische Trends, welche auch die muskuloskelettale Chirurgie beeinflussen:

\begin{abstract}
Die minimalinvasive Chirurgie hat ein hohes Potenzial, Sekundärtrauma zu minimieren und damit den Krankenhausaufenthalt zu verkürzen oder den Patienten schneller zu rehabilitieren und auch Sekundärschäden zu vermeiden (Abb. 1).
\end{abstract}

OP-JOURNAL 2014; 30: 188-195

(c) Georg Thieme Verlag KG Stuttgart · New York DOI http://dx.doi.org/10.1055/s-0034-1383340
- Laut der SMISS [1] kann die durchschnittliche Aufenthaltsdauer bei lumbaler Wirbelkörperfusion von 5-7 Tagen (offener Eingriff) auf 2-3 Tage (minimalinvasiver Eingriff) reduziert werden. Die wesentliche Herausforderung ist neben der komplexeren Vorgehensweise die fehlende direkte Visualisierung des Situs und damit die Notwendigkeit eines bildgestützten Verfahrens.

- Trend zu der Einführung von integriertem Qualitätsmanagement: Ein integriertes Qualitätsmanagement hat auch in der Chirurgie ein hohes Potenzial, zum einen die Behandlungsergebnisse zu verbessern, aber auch die Kosten der Behandlung zu reduzieren.

Eine Kostenreduzierung kann sowohl aufgrund niedrigerer Revisions- oder Komplikationsraten als auch der Reduzierung der Primärkosten aufgrund effizienterer Behandlungsabläufe erzielt werden. Ein wesentliches Element eines Qualitätsmanagementsystems ist die integrierte und lückenlose Dokumentation aller Eingriffe, um dem Prinzip Plan $\rightarrow$ Do $\rightarrow$ Check $\rightarrow$ Act folgend eine kontinuierliche Verbesserung und Weiterentwicklung des gesamten Behandlungsablaufs zu ermöglichen (Abb.2). Die Bildgebung, präoperativ, intraoperativ wie auch postoperativ, spielt hier eine wesentliche Rolle, um die Behandlung zu dokumentieren und aufgrund der Bilder zu überprüfen, inwieweit das operative Ergebnis der Planung entspricht, um bei evtl. Abweichungen frühzeitig korrigierend einzugreifen und Maßnahmen zu identifizieren und um zukünftige Eingriffe zu verbessern.

- Ein solcher kontinuierlicher Verbesserungsprozess auf Basis eines Qualitätsmanagementsystems macht dann auch eine Standardisierung der Behandlungsabläufe notwendig, um den hohen Qualitätsstandard dann auch reproduzierbar zu machen. Sowohl die Schulung des ärztlichen Personals als auch die Verfügbarkeit von optimierten Werkzeugen profitieren erheblich von einer Standardisierung der Behandlungsabläufe und führen damit zu besseren Behandlungsergebnissen bei geringeren Kosten. Die Bildgebung kann hier einen wesentlichen Beitrag zur Standardisierung liefern, da mithilfe von bildbasierter Planung und bildunterstützter Chirurgie der Eingriff in hohem Maße reproduzierbar werden kann.

- Der Kostendruck auf die Gesundheitssysteme betrifft auch die operativen Eingriffe. Aufgrund des hohen Kostenanteils der Chirurgie in den Krankenhäusern von bis zu 33\% [2], steht auch diese im Fokus der Einsparungen. Neben dem Potenzial zur Reduzierung von Sekundärkosten wie Revisionsraten kann die Bildgebung auch einen wesentlichen Beitrag zur Reduzierung 

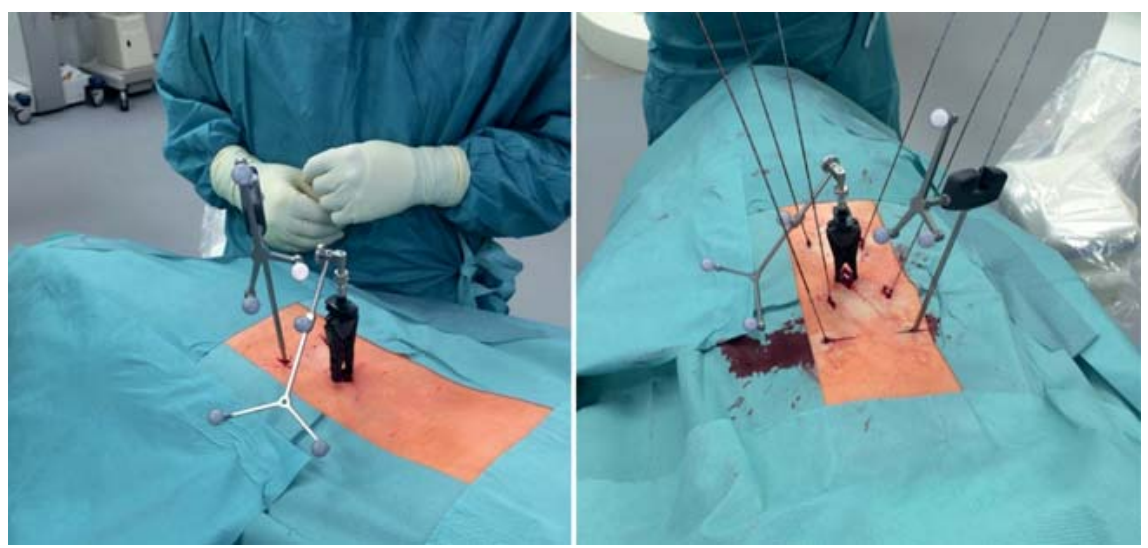

Abb. 1 Minimalinvasiver Eingriff.
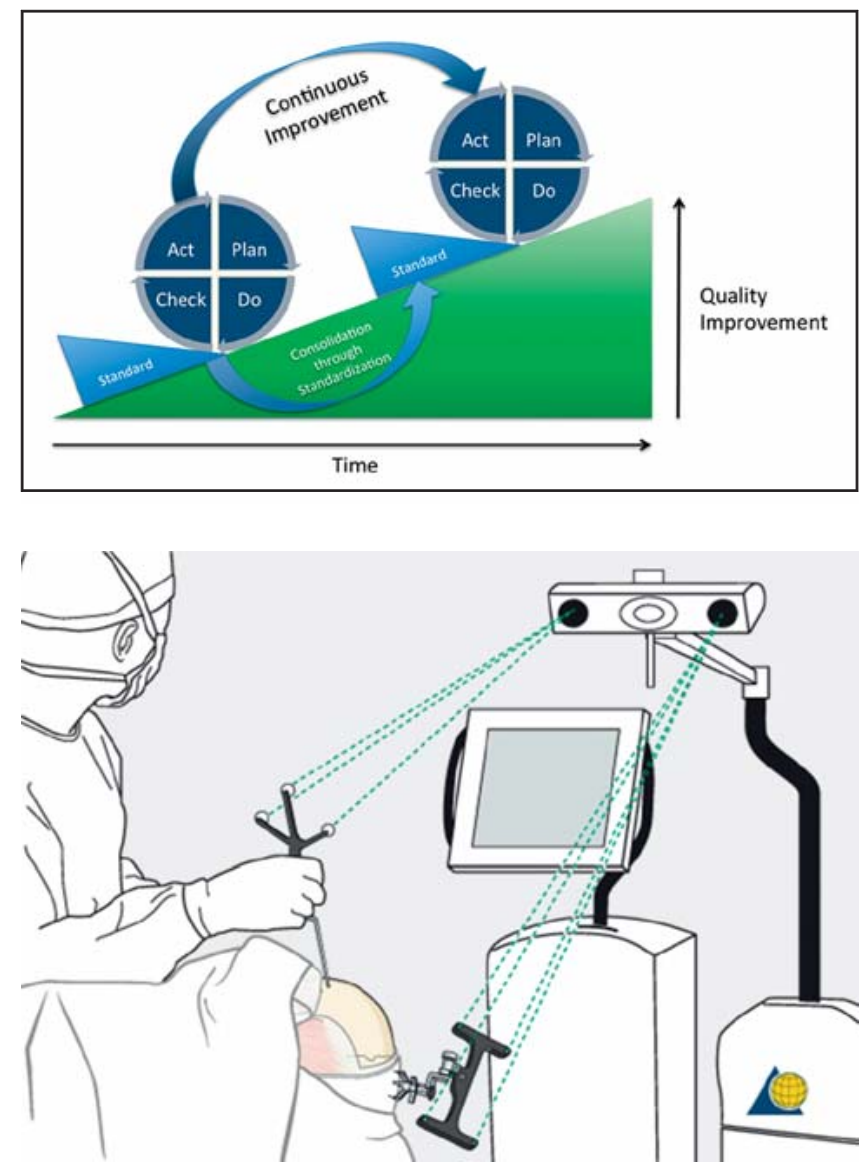

von OP-Zeiten und damit zu entsprechender Kostenreduzierung führen.

Damit spielen Bildgebung und bildgestützte Navigationsverfahren eine zentrale Rolle, um die Trends in der Chirurgie zu unterstützen. Die heute im Einsatz befindlichen Technologien zur Bildgebung und Navigation ermöglichen das bereits, haben aber Limitierungen bez. der Einsatzbreite. Daher ist eine Weiterentwicklung der Technologien essenziell, um die o.g. Trends in vollem Um-
Abb. 3 Line-ofSight-Problem. (c) AO Foundation,

Switzerland.

Abb. 2 Kontinuierlicher Verbesserungsprozess als Bestandteil des Qualitätsmanagements [3].

fang zu unterstützen und damit die entsprechenden Veränderungen zu ermöglichen.

\section{Limitierung heutiger Bildgebungs- und Navigationsverfahren}

\section{Intraoperative Bilder sind oft 2-dimensional}

Die intraoperative Bildgebung, meist über Einsatz des mobilen C-Bogens, ist i.d.R. ein 2-D-Bildgebungsverfahren.
Chirurgische Eingriffe sind jedoch 3-dimensionale Problemstellungen. Daher muss der Chirurg die 2-dimensionalen Bilder in sein 3-dimensionales Problem „übersetzen“, was insbesondere bei komplexen Strukturen zu Fehlinterpretationen der 2-dimensionalen Bilder führen kann.

Der Einsatz von Bildgebung ist nicht optimal in den Workflow integriert

- Obwohl mit dem chirurgischen C-Bogen bereits vor vielen Jahren ein dediziertes Röntgensystem für den OP eingeführt wurde, besteht immer noch Verbesserungspotenzial hinsichtlich eines optimierten Einsatzes im OP. So kann z.B. die händische Positionierung des C-Bogens, wegen missverständlicher Kommunikation zwischen dem Chirurgen und dem Bedienpersonal oder schwieriger Reproduzierbarkeit, zu langwierigen Trial-and-Error-Prozessen bei Aufnahmen im OP führen. Bei den gängigen, optischen Navigationssystemen ist ein häufiges Problem die erforderliche Line of Sight, die es notwendig macht, dass die Navigationskamera einen direkten Blick auf die navigierten Instrumente haben muss und damit die Position des OP-Personals und sonstiger Geräte einschränkt (Abb.3). Eine alternative Navigationstechnologie ist das elektromagnetische Tracking, welches keine Line of Sight erforderlich macht und damit auch eine bessere Integration der Marker in die Werkzeuge ermöglicht. Diese Technologie ist jedoch sehr störanfällig gegenüber elektromagnetischem Rauschen, wie z.B. von Bohrmaschinen erzeugt. Daher hat sich der Einsatz dieser Technologie, insbesondere in der Unfallchirurgie, nicht etabliert.

- Die Bildgebungs- und Navigationssysteme eröffnen aufgrund der Digitalisierung und fortschreitenden Computerisierung immer neue Möglichkeiten, die jedoch die Anwendung mitunter komplexer machen. Des Weiteren erfordern die verschiedenen Systeme i.d.R. eine eigene Bedienkonsole mit unterschiedlichen Bedienkonzepten, was die Komplexität erhöht. Die damit verbundene Komplexität in der Anwendung kann trotz offensichtlicher Vorteile die Verbreitung limitieren, da diese ein hohes Maß an Expertenwissen erfordern kann.

- Die präoperative Bildgebung könnte besser im intraoperativen Prozess genutzt werden. Moderne, hochauf- 
lösende CTs und MRTs bieten sehr gute Informationen, nicht nur für die Diagnose oder Planung des Eingriffs, die Aufnahmen könnten auch wertvolle Hilfestellungen für den intraoperativen Prozess liefern. Ein Beispiel ist die Vermeidung von Wrong Level Surgery bei Wirbelsäulenoperationen: mithilfe des präoperativen CTs oder MRTs wird die Diagnose und damit die Entscheidung, an welchem Wirbelkörper operiert werden soll, getroffen. Leider kann diese Bildinformation bisher nicht verwendet werden, um intraoperativ schnell und sicher den $\mathrm{zu}$ operierenden Wirbelkörper zu identifizieren.

\section{Es fehlen Werkzeuge, um die Planung umzusetzen}

Neue, intraoperative Planungstechnologien wie syngo.iGuide der Fa. Siemens ermöglichen es, sehr genau einen Eingriff wie das Setzen von Schrauben zu planen. Oftmals ist dann jedoch die Umsetzungsgenauigkeit der limitierende Faktor, da die von Hand geführten Instrumente aufgrund der fehlenden Positionsmessung oder manuellen Führung in ihrer Positioniergenauigkeit limitiert sind.

Ein erhöhter Einsatz von röntgenbasierter Bildgebung macht ein besseres Strahlungsmanagement erforderlich

Bildgestützte Verfahren machen einen erhöhten Einsatz der Bildgebung im OP erforderlich. Auf absehbare Zeit bleibt die röntgenbasierte Bildgebung das Mittel der Wahl in der intraoperativen Bildgebung, insbesondere für muskuloskelettale Eingriffe. Um zu vermeiden, dass das OP-Personal damit einer höheren Strahlenbelastung ausgesetzt wird, sind neue und verbesserte Verfahren zum Strahlungsmanagement erforderlich.

Im folgenden Kapitel werden nun Technologien und Verfahren beschrieben, welche die o.g. Limitierungen auflösen können und damit die Zukunft der Bildgebung und Navigation gestalten werden.

\section{Technologien der Zukunft für die integrierte Bildgebung und Navigation}

\section{Intraoperative 3-D-Bildgebung}

- Intraoperative 3-D-Bildgebungssysteme (3-D-C-Arm, Hybrid-OP (Abb. 5), intraoperatives $\mathrm{CT}$, MRT):

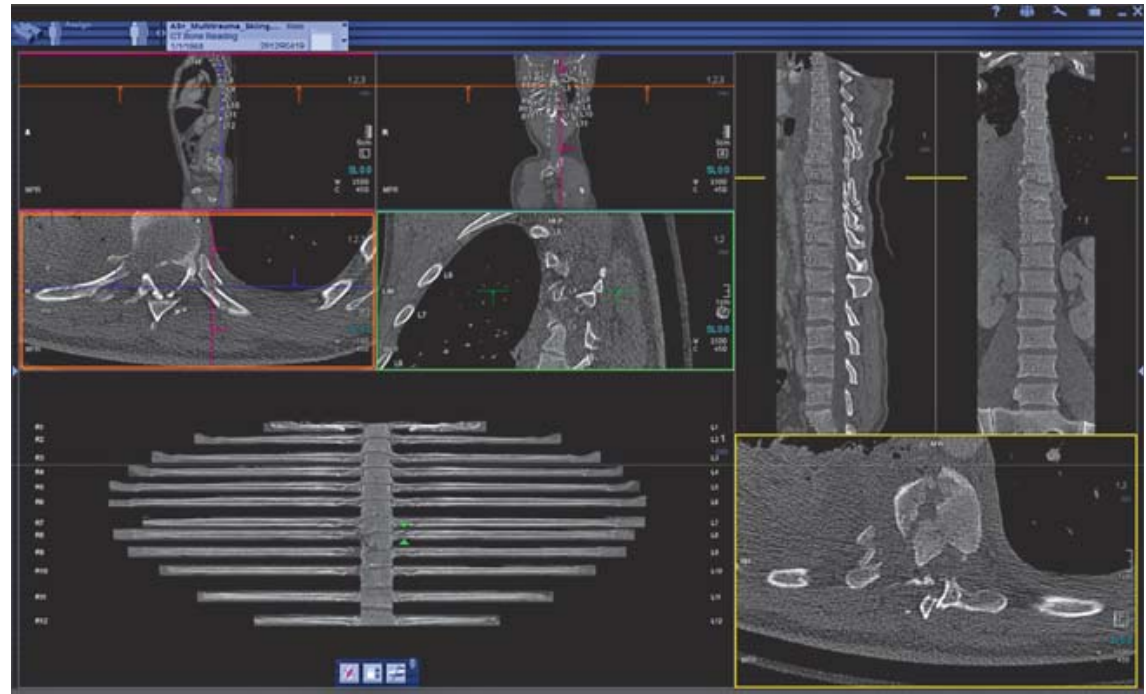

Abb. 4 Die CT Bone Reader Application von syngo.via der Siemens AG bezeichnet automatisch die Rippen und Wirbelkörper.

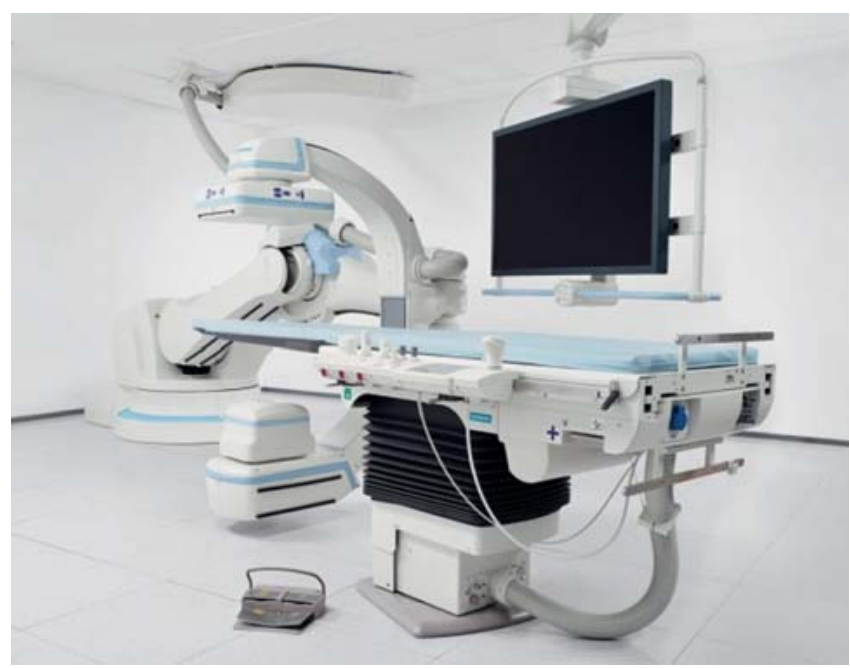

Abb. 5 Artis zeego der Fa. Siemens. Mit freundlicher Genehmigung der Siemens AG.

Mithilfe der Motorisierung eines C-Bogens und eines speziellen Aufnahmemodus kann dieser durch viele Aufnahmen mit unterschiedlichen Projektionen bei niedriger Dosis ein 3-D-Bild rekonstruieren.

- Mit diesen 3-D-Bildern lassen sich komplexe Anatomien intraoperativ besser visualisieren oder diese Aufnahmen zum bildgestützten Eingriff, der intraoperativen Kontrolle zur Vermeidung von Revisionen oder auch zur Navigation verwenden.

- Werkzeuge, um aus 2-D-Bildern relevante 3-D-Informationen $\mathrm{zu}$ berechnen und zu visualisieren: Bei bekannten Strukturen (z.B. ein Implantat oder gewisse im Röntgenbild sichtbare und geometrisch bekannte Strukturen) lässt sich aus der 2-D-Aufnah- me die 3-D-Position des Objekts berechnen und entsprechend in das Röntgenbild einblenden. Mit dieser Technologie kann man dann besser die Lage des Objekts im 3-D-Raum beurteilen und damit z.B. Position und Länge der Schrauben besser planen auf Basis des 2-D-Bildes. Ein Beispiel hierzu ist die $\mathrm{X}$-in-One-Technologie des AO Research Institutes [4], mit der z.B. für die proximale Humerusverriegelung oder proximale Femurnagelverriegelung die Position und Länge der Schrauben genauer bestimmt werden kann (Abb. 7 und 8).

\section{Workflow-integrierte Bildgebungssysteme}

\footnotetext{
- Robotisierte C-Bögen: Hybrid-OP Artis zeego (Abb. 5), neuartige mobile C-Bögen:
} 


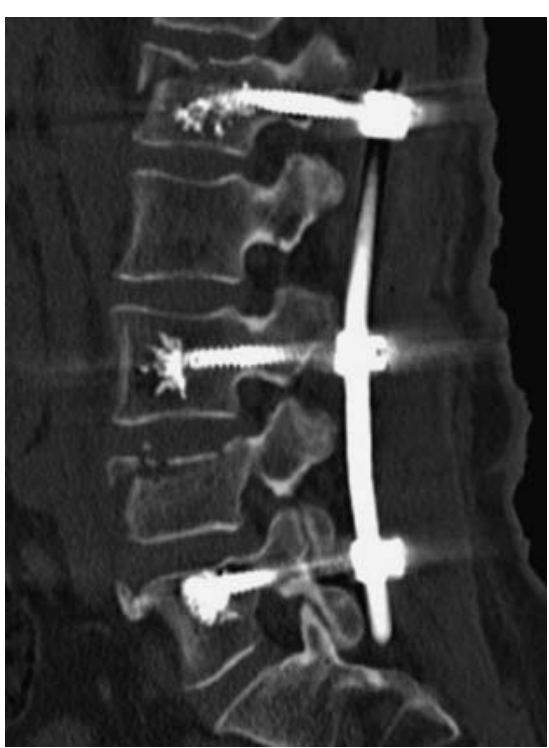

Abb. 6 3-D-Bild der Wirbelsäule, aufgenommen mit Artis zeego.

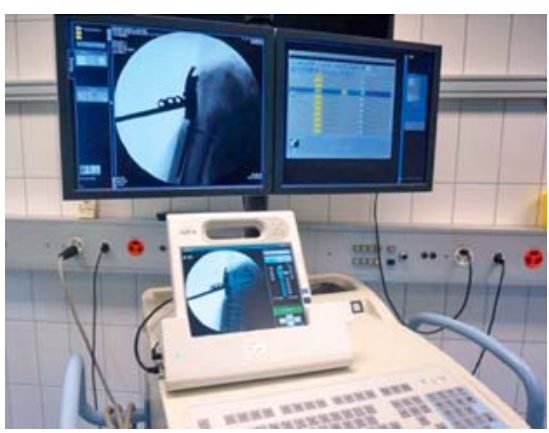

Abb. 7 Medizinischer Tablet-Computer mit $X$-in-One-Software in Verbindung mit einem C-Bogen. Mit freundlicher Genehmigung des AO Research Institutes Davos.

Mithilfe der Motorisierung der C-Bögen lassen sich Positionen motorisch genau anfahren.

Damit ist es möglich, vorherige Positionen wieder exakt anzufahren oder auf Basis von vorherigen 3-D-Aufnahmen spezielle Aufnahmen im 3-D-Bild zu planen und die dazu notwendige Position dann automatisch anzufahren. Diese Technologie kann daher zu einer erheblichen Dosis- und Zeiteinsparung führen. Ein Beispiel für eine solche Vorgehensweise ist das System syngo.iGuide, welches z. B. für die Vertebroplastie oder die Wirbelkörperverschraubung eingesetzt werden kann, und eine bildgestützte Planung mit einer C-Bogen-Automatisierung verbindet.

Das System syngo.iGuide ermöglicht, nadelbasierte Prozeduren wie z.B. Vertebroplastien, Lumbalpunkturen, Biopsien oder Radiofrequenzablationen präziser und effektiver als ohne Nutzung

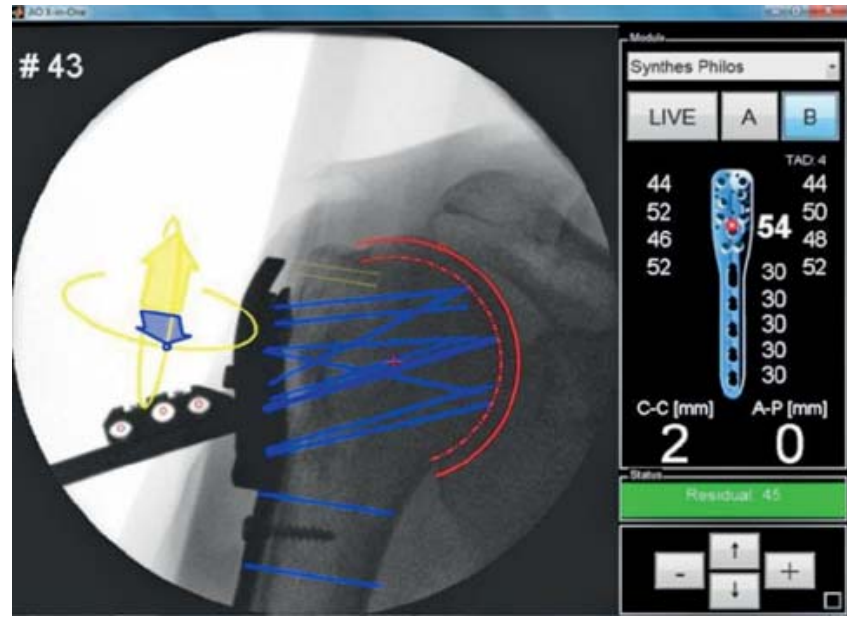

Abb. 8 Software Screenshot. Die Trajektorien der Schrauben einer PHILOSPlatte (blaue Linien) werden in das Röntgenbild projiziert. Mit freundlicher Genehmigung des AO Research Institutes Davos.

dieser Technologie durchzuführen. Der intuitive Workflow zeigt Länge und Angulation des Nadelpfads an und positioniert den C-Bogen automatisch so, dass mithilfe des integrierten Laserpointers die Hauteintrittsstelle angezeigt wird und der Nadelvorschub unter Fluroskopiekontrolle flüssig durchgeführt werden kann (Abb. 9 bis 13).

\section{- Integriertes Bediensystem für alle Bildinformationen: Ein integriertes Bediensystem sollte patientenzent- riert alle notwendigen Informationen (geräte- und prozessübergreifend) darstellen (Abb. 14, 15 und 18).}

Des Weiteren muss die angezeigte Information Workflow-getrieben sein, d.h. die in dem entsprechenden WorkflowSchritt notwendigen Bildinformationen sollen möglichst automatisch so dargestellt werden, wie sie für den entsprechenden Workflow-Schritt benötigt werden. Ein Beispiel hierfür wäre ein System zur automatischen Detektion von anatomischen Objekten oder Implantaten, welches die multiplanaren Rekonstruktionen (MPR) der 3-D-Bilddaten so berechnet, dass die Schnittbilder der relevanten Anatomie den Implantatlagen entsprechen. Ein Beispiel hierfür ist der CT Bone Reader von syngo.via der Fa. Siemens (Abb. 4).

- Verwendung von präoperativen Bilddaten für intraoperative Führung: Hierzu ist eine Registrierung der präoperativen Bilddaten in das intraoperative Szenario notwendig. Dies kann z.B. durch die Registrierung und damit Überlagerung eines präoperativen CTs mit einem intraoperativen C-BogenBild erfolgen, um damit in der 2-D-CBogen-Aufnahme den zu operierenden Wirbelkörper zu identifizieren. Dazu wird zuerst präoperativ im CT-
Bild der zu operierende Wirbelkörper durch eine Annotation des Bildes markiert. Im OP wird vor Schnitt eine CBogen-Aufnahme der Region gemacht, in der der zu operierende Wirbelkörper liegt. Die Aufnahme kann eine laterale, a.-p. oder kombinierte Aufnahme sein. Die entsprechende Software überlagert dann automatisch das Röntgenbild mit dem präoperativen, markierten CT-Bild, sodass in dem Röntgenbild die Markierung des Wirbelkörpers sichtbar wird und damit dieses zur Lokalisierung des Wirbelkörpers verwendet werden kann (Abb. 16) [5,6].

\section{Navigation und robotische Assistenzsysteme}

Die oben beschriebenen, genaueren Planungsmöglichkeiten aufgrund von 3-DTechnologie erfordern nun auch Technologien, um die Planung exakt umsetzen zu können. Die Navigationstechnologien sind Verfahren, um dies möglichst strahlungsfrei zu ermöglichen, indem die Instrumentenposition in Echtzeit gemessen wird und diese in das 2-D- oder 3-D-Röntgenbild eingeblendet wird. Eine gleichzeitige Einblendung der Planung erlaubt es nun, das Instrument entsprechend der Planung zu führen. Heute besteht das Messsystem für Navigationssysteme meist aus einer optischen Kamera, welche die Position der Instrumente, die mit speziellen optisch reflektierenden Markern ausgestattet sind, misst und dann das Instrument positionsgenau und in Echtzeit in das Bild einblendet.

In der Forschung werden auch Technologien wie Gyroskope oder auch RFID (radio-frequency identification) für die Navigation eingesetzt. 


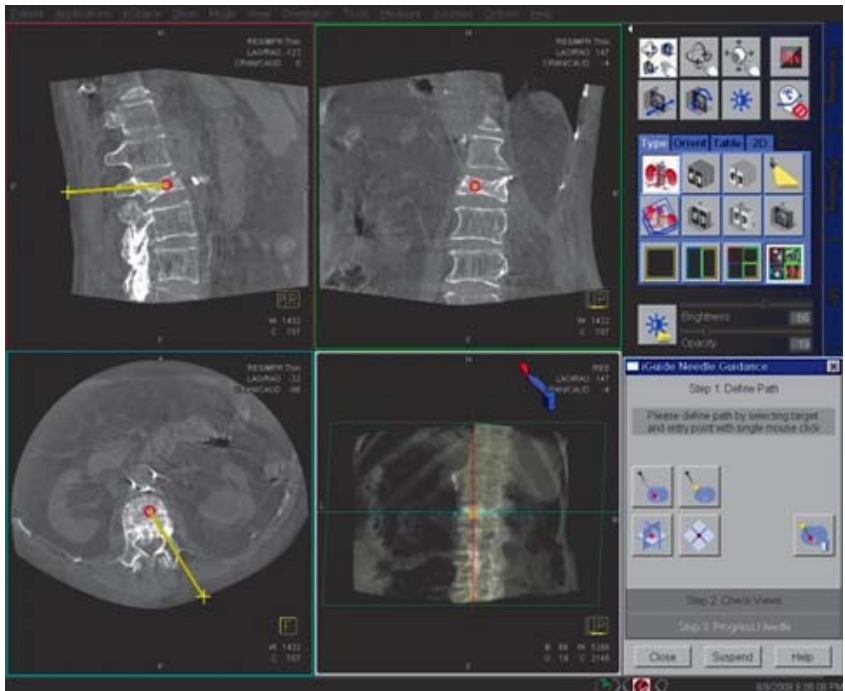

Abb.9 Planung des Nadelpfads. Mit freundlicher Genehmigung der Siemens AG.

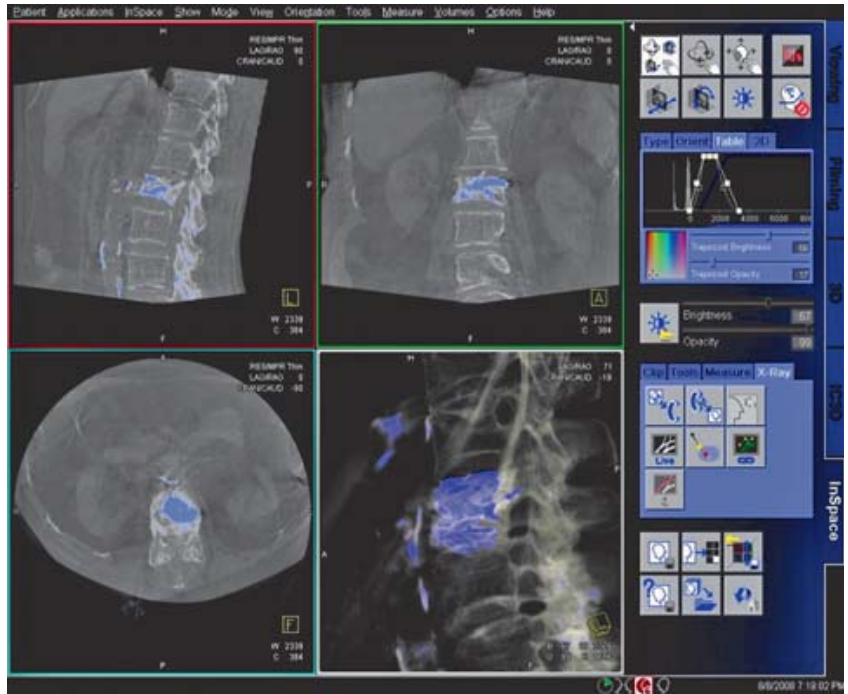

Abb. 13 Finaler Check des Ergebnisses mit syngo.DynaCT. Mit freundlicher Genehmigung der Siemens AG.
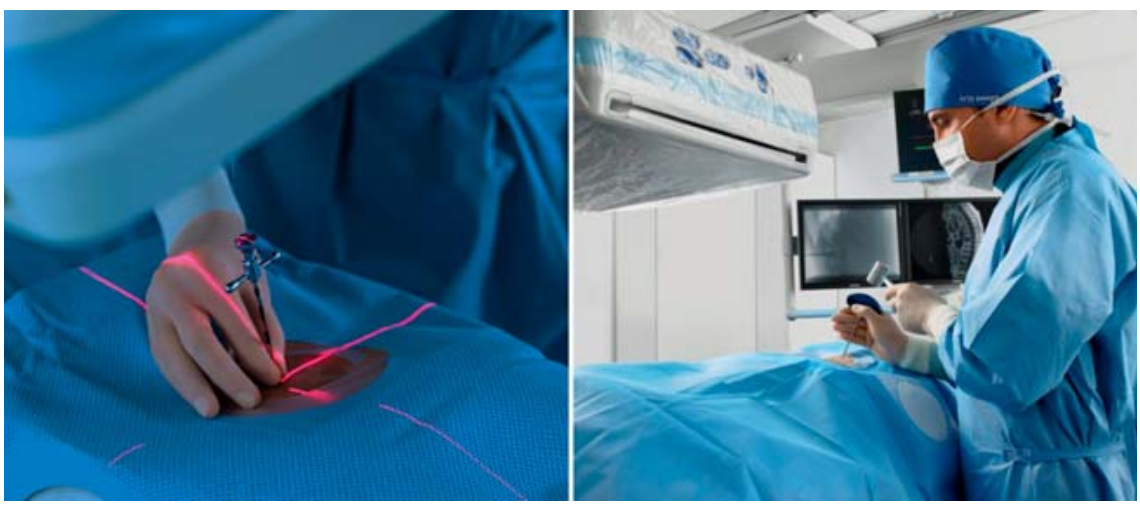

Abb. 11 Hauteintrittspunkt mithilfe des integrierten Lasers festlegen. Mit freundlicher Genehmigung der Siemens AG.

Ob und wie sich diese Technologien für die chirurgische Navigation etablieren werden, hängt davon ab, inwieweit sie die erforderlichen Genauigkeiten erreichen, robust genug gegenüber Störungen sind und Probleme der etablierten Technologie lösen können (wie z.B. Line of Sight mit optischen Verfahren).

Neue robotische Technologien, wie der Leichtbauroboter (LBR) der Fa. KUKA Labs, haben die Roboter signifikant verkleinert, was ein wesentliches Kriterium für Anwendungen im $\mathrm{OP}$ ist (Abb. 17).

Ein solcher Roboter kann zur Positionierung des chirurgischen Instruments dienen. In Verbindung mit dem Bildgebungssystem kann nun das Instrument exakt positioniert werden, sodass sich der Chirurg, robotisch unterstützt, auf die Führung des Instruments fokussieren kann. Entsprechende Systeme für spezielle Anwendungen gibt es heute
z.B. von der Fa. Mazor für Pedikelverschraubungen.

\section{Integriertes Dosismanagementsystem}

Der verstärkte Einsatz von röntgenbasierter Bildgebung im OP macht es notwendig, Personal und Patienten besser vor Strahlung zu schützen.

Dazu sind 3 Maßnahmen erforderlich:

- Schulung über die Wirkung von Strahlung sowie Vermeidung und Reduzierung der Strahlenbelastung

- Verbesserung und konsequenter Einsatz von Schutzmaßnahmen

- Minimierung der eingesetzten Strahlung

Die Wirkung von Röntgenstrahlen folgt physikalischen Prinzipien, deren grundlegendes Verständnis notwendig ist, um den Patienten und das OP-Personal entsprechend vor Strahlung zu schützen.

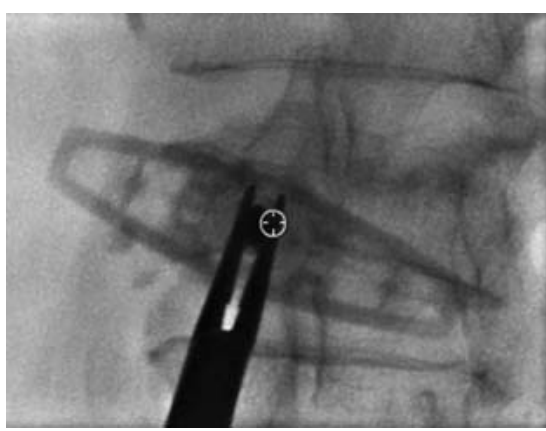

Abb. 10 Bull's Eye View. Mit freundlicher Genehmigung der Siemens AG.

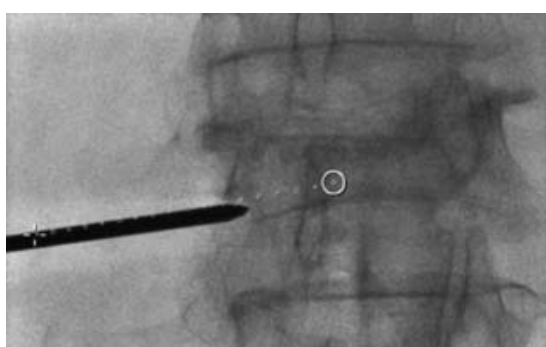

Abb. 12 Geplanter Pfad wird unter Fluoroskopie angezeigt. Mit freundlicher Genehmigung der Siemens AG.

Daher ist eine konsequente, rollenbasierte Schulung zu Strahlenschutz aller Personen, die im OP arbeiten, notwendig. Des Weiteren helfen moderne Dosismesssysteme, die Strahlendosis auch in Echtzeit zu überwachen, sodass man während des Eingriffs die Strahlenexposition darstellen und sein Verhalten entsprechend anpassen kann. Robotische Systeme ermöglichen es, die Strahlenexposition bei strahlungsintensiven Arbeitsschritten zu reduzieren oder sogar 


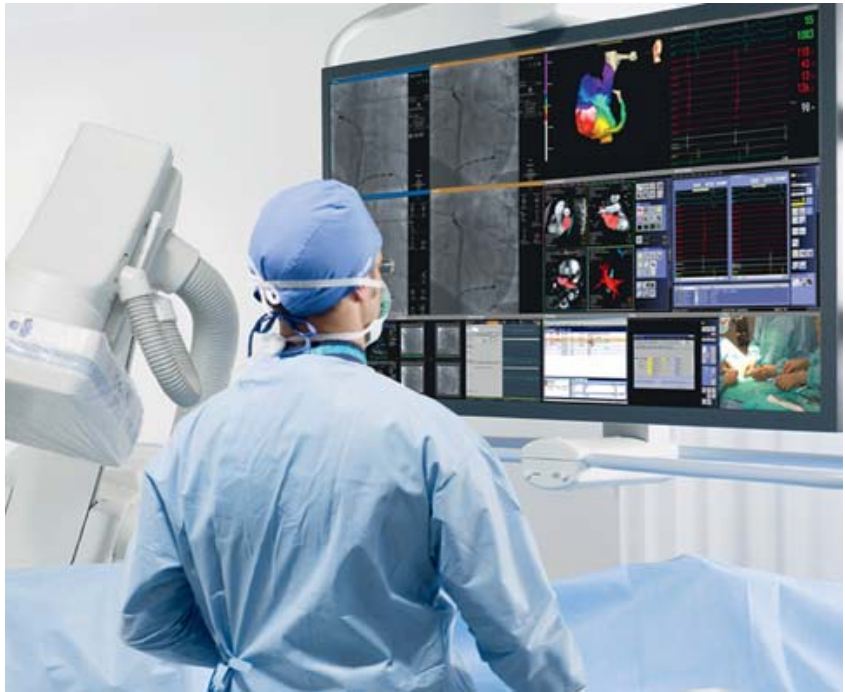

Abb. 14 Das multimodale Display des Artis zeego kann auf einem Bildschirm in konfigurierbarem Layout Informationen, Bilder und Videos verschiedener Modalitäten darstellen. Mit freundlicher Genehmigung der Siemens AG.

auszuschließen, da der Operateur den entsprechenden Arbeitsschritt aus sicherer Entfernung durchführen kann. Ein Beispiel ist der motorisierte C-Bogen Cios Alpha, der es ermöglicht, 2 C-Bogen-Positionen zu speichern und diese dann auf Knopfdruck wieder anzufahren und damit ohne Durchleuchtungskontrolle eine vorherige Projektion wieder einzustellen.

Navigationstechnologien helfen, gewisse bildgeführte Arbeitsschritte ganz ohne
Strahlenexposition durchzuführen, da die Instrumente in ein vorher aufgenommenes Bild in Echtzeit eingeblendet werden und damit während des entsprechenden Arbeitsschritts keine Strahlung notwendig ist. Weitere IGS-Technologien (IGS: Image Guided Surgery) wie intraoperative Planungssysteme (z.B. syngo.iGuide), das Einblenden von Trajektorien der Instrumente, wie z.B. des K-Drahtes oder die ortsgenaue Überlagerung eines Röntgenbilds mit einem Videobild in Echtzeit, ermöglichen die

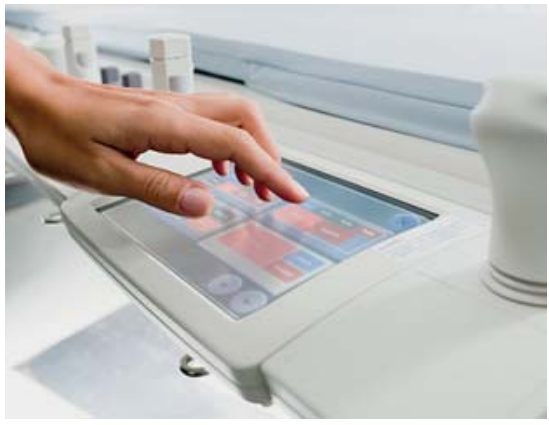

Abb. 15 Die tischnahe Bedienung des Artis zeego mit Touchpanel und Joysticks ermöglicht die Bedienung des Systems direkt vom OP-Tisch aus. Mit freundlicher Genehmigung der Siemens AG.

Vermeidung von Strahlung bei Arbeitsschritten, zu denen der Operateur die Bildinformation benötigt. Neue Röntgentechnologien wie z.B. der Flachdetektor ermöglichen es, bei gleicher Bildqualität eine geringere Dosis zu verwenden. $\mathrm{Zu}$ diesen Basistechnologien wird es weitere Fortschritte auch bei der Röntgenstrahlerzeugung geben.

\section{Ausblick: der OP der Zukunft}

Viele der im vorherigen Kapitel genannten Technologien werden für den OP der Zukunft eine wichtige Rolle spielen, aber

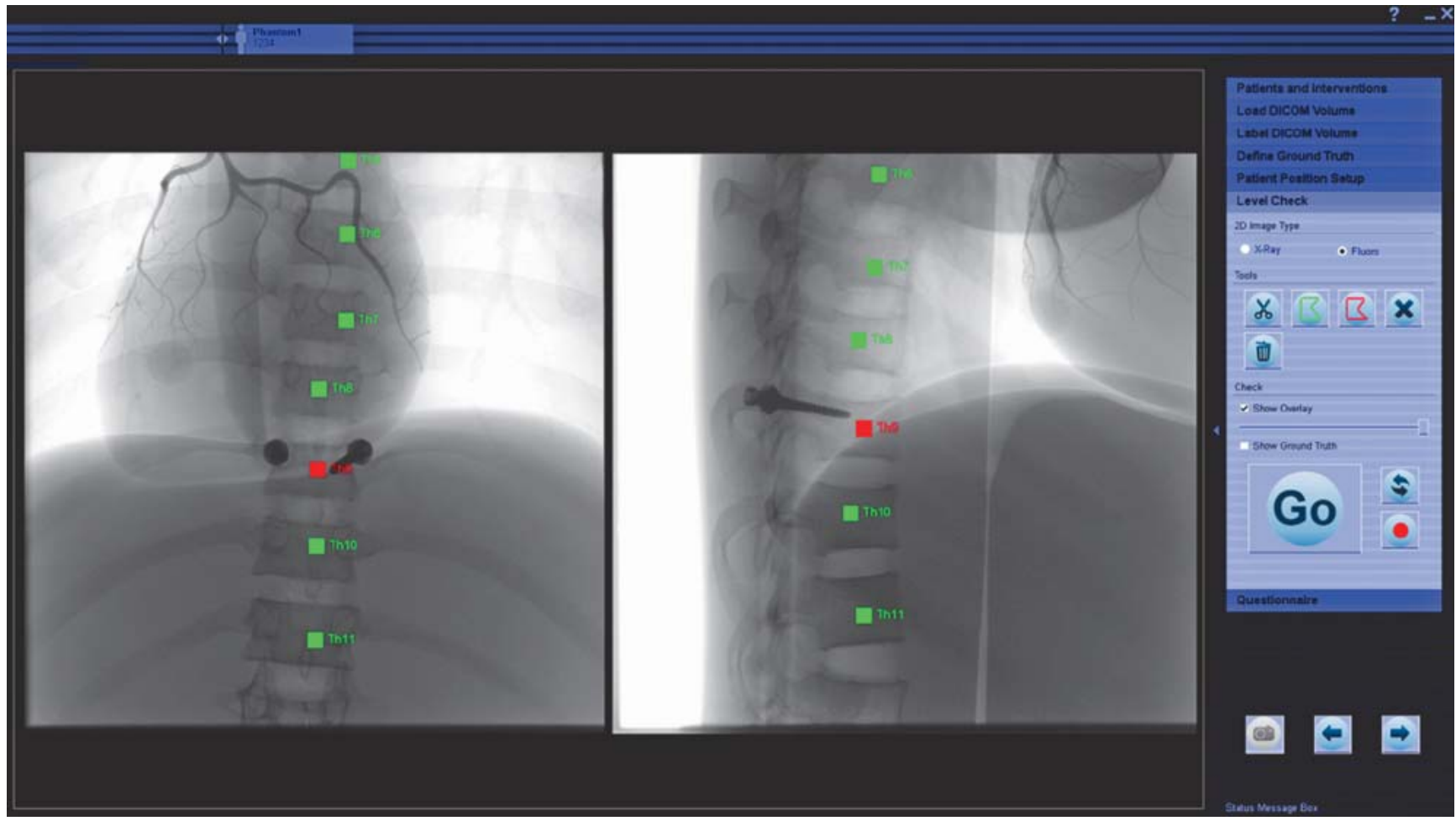

Abb. 16 Die automatische Registrierung einer intraoperativen C-Bogen-Aufnahme mit dem präoperativen CT-Bild, in welchem präoperativ der relevante Wirbelkörper markiert wurde, ermöglicht die schnelle und sichere Identifizierung des relevanten Wirbelkörpers. Mit freundlicher Genehmigung der Fa. Siemens. 


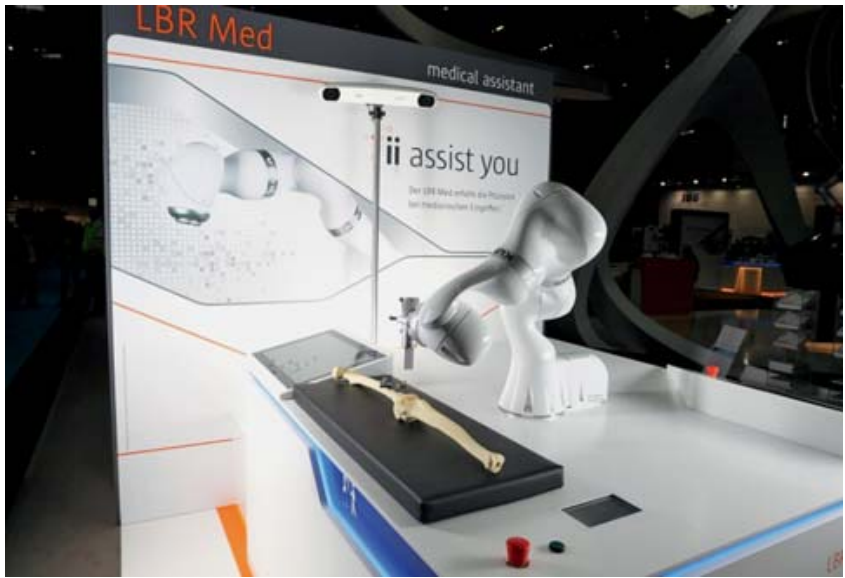

Abb. 17 Leichtbauroboter (LBR Med) der Fa. KUKA Labs. Mit freundlicher Genehmigung der Fa. KUKA Laboratories (www.kuka-labs.com).

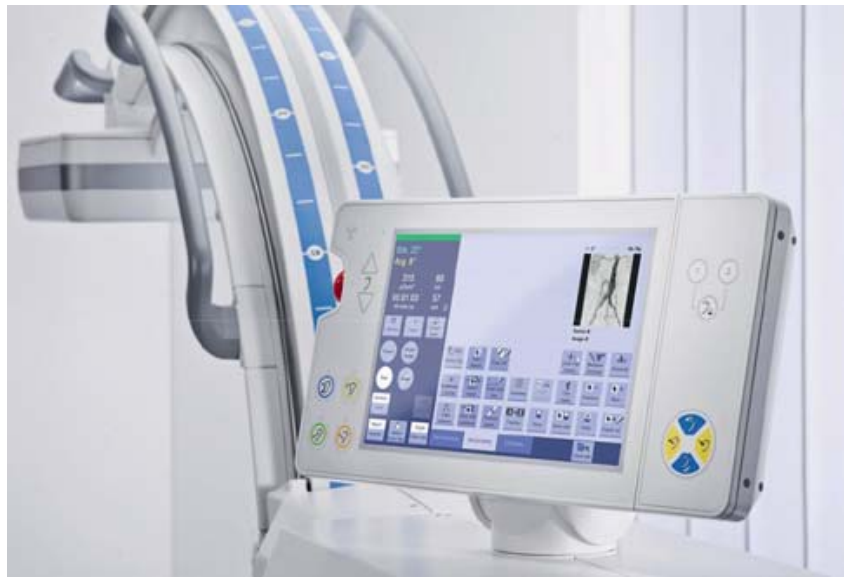

Abb. 18 Die Bedienung des Cios Alpha ist über das gleiche Bedienpanel vom C-Bogen, vom Trolley und vom OP-Tisch möglich. Mit freundlicher Genehmigung der Siemens AG. nicht alle werden sich durchsetzen. Entscheidend wird dabei sein,

- inwieweit die Technologien sich in ein technologisches Gesamtkonzept integrieren,

- diese sich in den chirurgischen Workflow integrieren und nicht umgekehrt und

- neue Bedienkonzepte eingeführt werden, welche sich weitestgehend automatisch am chirurgischen Workflow orientieren.

Im Folgenden eine schematische Visionsskizze, wie der OP der Zukunft aussehen könnte (Abb. 19 und 20). Bei aller „visionärer Kreativität" muss der OP der Zukunft Qualitätsverbesserungen bei gleichzeitiger Kostenreduzierung und Risikominimierung für das Personal ermöglichen. Dabei müssen stets der Patient und dessen Wohl im Zentrum aller Handlungen stehen.

Im OP der Zukunft werden die chirurgischen Eingriffe im Wesentlichen bildgestützt und robotisch durchgeführt.

Die OP-Schwester arbeitet patientennah und ist für die wesentlichen Tätigkeiten am Patienten zuständig. Über robotische Arme werden sowohl die Instrumente geführt als auch die Bildgebung wie Röntgen, Ultraschall oder Endoskopie gesteuert. Neuartige Oberflächen vereinfachen das Sterilitätsmanagement. Der Operateur arbeitet von einer Bedienkonsole aus (Abb. 20). Von dort hat er Zugriff auf die Bedienung der robotisch geführten Instrumente wie auch die Bildgebung. Über entsprechende IT-Systeme hat er kontextspezifisch Zugriff auf alle relevanten Informationen und kann

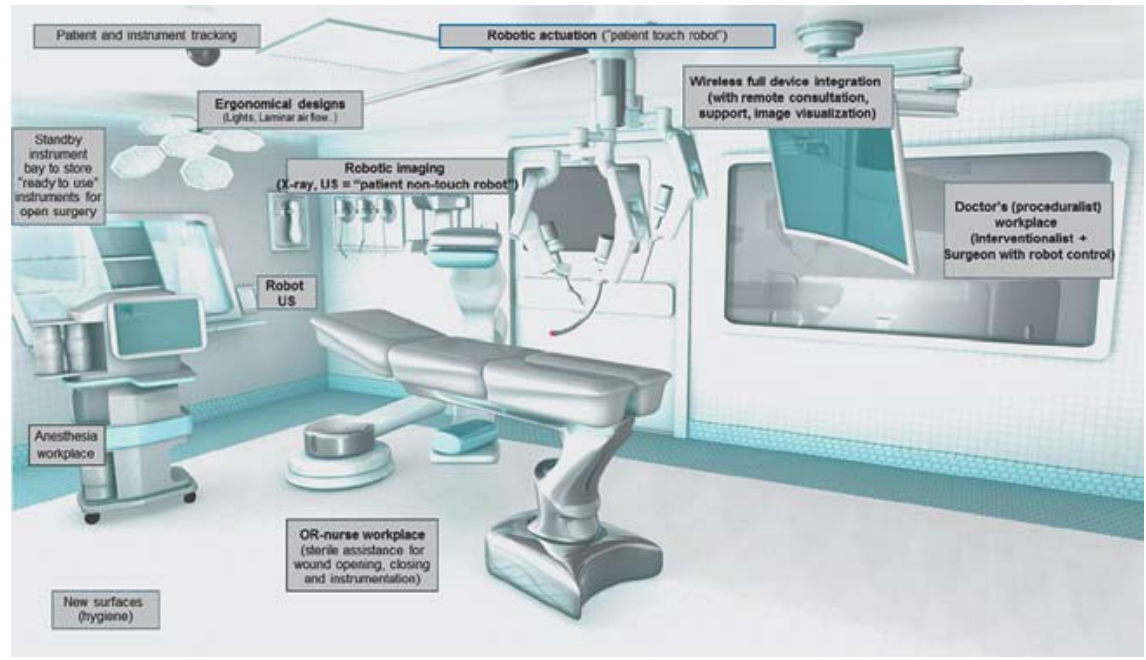

Abb. 19 Ein Szenario für den OP der Zukunft. Mit freundlicher Genehmigung der Siemens AG.

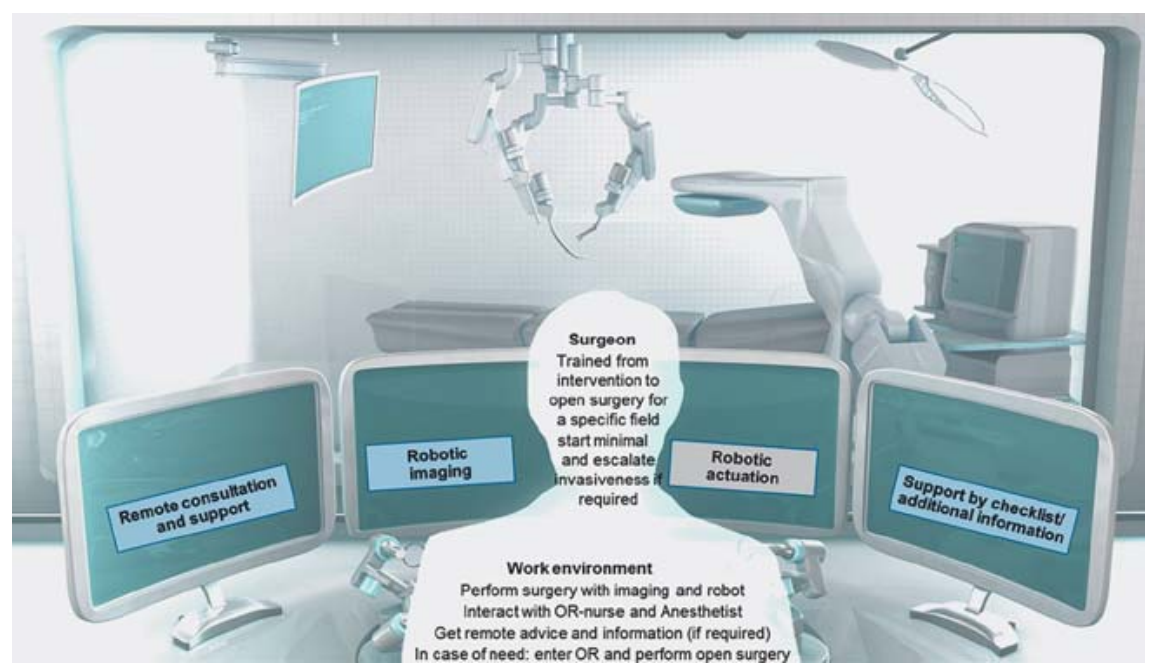

Abb. 20 Arbeitsplatz des Chirurgen im OP der Zukunft. Mit freundlicher Genehmigung der Siemens AG.

Jochen Dick, Florian Gebhard: Zukunft der intraoperativen Bildgebung und Navigation 
Experten und andere Drittpersonen ferngesteuert in die Operation mit einbinden.

\section{Literatur}

${ }^{1}$ Society for Minimally Invasive Spine Surgery. Frequently asked questions about minimally invasive spine surgery. Im Internet: http:// www.smiss.org/minimally-invasive-faq.php; Stand: 11.11.2014

2 Heuser D. Anforderungen an das System OP Arbeitszeitmodelle - Optimierung von Ablaufprozessen durch interdisziplinäres Personalmanagement. Zentralbl Chir 2001; 126 : 337-339

${ }^{3}$ Vietze J. Depiction of the Deming cycle (17.06.2013). Im Internet: http://commons.
wikimedia.org/wiki/File:PDCA_Process.png; Stand: 11.11.2014

${ }^{4}$ AO Research Institute. Activity report 2013. Im Internet: https://www.aofoundation.org/ Structure/research/exploratory-applied-research/research-institute/publication-activity-repport/Pages/activity_reports.aspx;

Stand: 11.11.2014

${ }^{5}$ Otake Y, Wang AS, Stayman JW et al. Robust 3D-2D image registration: application to spine interventions and vertebral labeling in the presence of anatomical deformation. Phys Med Biol 2013; 58: 8535-8553

${ }^{6}$ Otake Y, Wang AS, Stayman JW et al. Automatic localization of vertebral levels in C-arm fluoroscopy: Evaluation of the LevelCheck algorithm in a preclinical cadaver study with realistic tissue deformation. Int J Comput Assist Radiol Surg 2013; Proceedings of the CARS 2013 Conference

\section{Dr. Jochen Dick}

Vice President New Business

XRay Products

Siemens AG, Healthcare

Hartmannstraße 16

91050 Erlangen

jochen.dick@siemens.com

Univ. Prof. Dr. med. Florian Gebhard

Ärztlicher Direktor

Klinik für Unfall-, Hand-, Plastische und Wiederherstellungschirurgie

Universitätsklinikum Ulm

Albert-Einstein-Allee 23

89081 Ulm 